\title{
Review of: "Genomic diversity of Helicobacter pylori populations from different regions of the human stomach."
}

\author{
Guillermo Perez Perez ${ }^{1}$ \\ 1 NYU Langone Health
}

Potential competing interests: The author(s) declared that no potential competing interests exist.

The manuscript of Wilkinson et at entitled "Genomic diversity of Helicobacter pylori populations from different regions of the human stomach" reported the diversity between and within different regions of the human stomach by combining population deep sequencing with conventional sequencing of multiple single colonies of $\mathrm{H}$. pylori isolated from paired antrum and corpus biopsies along with single biopsies from one region of three patients.

The authors reported extensive allelic diversity within the different regions of patient's stomach. Te authors found that antrum and corpus populations from the same patients grouped together phylogenetically suggesting that most of the studied patients were initially infected with a single strain. In addition, single colonies from antrum and corpus of the same patients grouped in distinct clades indicating mechanisms for adaptation.

This is an excellent and well conducted study with a clear description of the materials and methods. The result section is clearly presented and the figure are very illustrative of the points addressed by the authors. The discussion and conclusions are related to the experimental work and the results obtained. In general I have very little to add to this paper.

Perhaps my only comments is related to the results observed in patient 565 that shoed substantial gene content differences between single colonies and the lack of clustering of antrum and corpus strains in patients 295 and 444, the authors mentioned some possible genetic changes or the infection with more of one H. pylori strain. However, the authors never indicated the age, gender and country of origin of those three patients that may explain those differences. 